\title{
An Unusual Association: Takayasu's Arteritis and Tubulointerstitial Nephritis and Uveitis Syndrome
}

\author{
Abir Derbel, Mariam Ghribi, Sameh Marzouk, Zouhir Bahloul \\ Service de Médicine Interne, CHU Hédi Chaker, Sfax, Tunisia
}

Received: 03/10/2020

Accepted: 07/10/2020

Published: $17 / 11 / 2020$

How to cite this article: Derbel A, Ghribi M, Marzouk S, Bahloul Z. An unusual association: Takaysu's arteritis and tubulointersitial nephritis and uveits syndrome. EJCRIM 2020;7: doi:10.12890/2020_002040.

Conflicts of Interests: The Authors declare that there are no competing interests.

Acknowledgements: This patient was supported by the Department of Internal Medicine guided by Dr Marzouk Sameh and Dr Derbel Abir. We thank the Ophthalmology Department for assistance and Professor Zouhir Bahloul for comments that greatly improved the manuscript.

This article is licensed under a Commons Attribution Non-Commercial 4.0 License

\section{ABSTRACT}

The coexistence of two immune-mediated diseases in the same patient is unusual. Takayasu's arteritis (TA), which is a chronic granulomatous vasculitis, was discovered in a 47-year-old woman followed for tubulointerstitial nephritis and uveitis syndrome (TINU syndrome). We present the first case of this association.

\section{LEARNING POINTS}

- Rare autoimmune diseases can coexist in the same patient.

- Physical examination is important so that coexisting diseases can be identified.

- Takayasu's arteritis was discovered as an incidental finding in a patient followed fortubulointerstitial nephritis and uveitis syndrome.

\section{KEYWORDS}

Longitudinally extensive transverse myelitis, LETM, spinodural arteriovenous fistula, SDAF, artery of Adamkiewicz

\section{INTRODUCTION}

Takayasu's arteritis (TA) is a chronic vasculitis of unknown aetiology which primarily affects the aorta and its main branches. It can be associated with autoimmune diseases such as rheumatoid arthritis ${ }^{[1]}$, inflammatory bowel disease ${ }^{[2]}$, ankylosing spondyloarthritis ${ }^{[3]}$, chronic thyroiditis ${ }^{[4]}$, granulomatosis and polyangiitis, which suggests that TA itself may also be an autoimmune disease ${ }^{[1]}$. To our knowledge, TA occurring in association with tubulointerstitial nephritis and uveitis syndrome (TINU syndrome) ha snot previously been described in the literature. We report the first case of this association.

\section{CASE DESCRIPTION}

A 47-year-old woman was admitted to hospital in 2008 for anterior uveitis of the right eye. She had no past medical history. She had experienced eye pain and redness for 15 days. She did not have fever or other symptoms. She had normal blood pressure of $130 / 80 \mathrm{mmHg}$ in both arms. There were no vesicularbreath sounds. Ophthalmological examination showed low visual acuity of $6 / 10$ in the right eye. Granulomatous keratic precipitates were evident on slit lamp examination. The left eye was normal. Biological analysis showed renal failure (creatinine $123 \mu \mathrm{mol} / \mathrm{I}$ ) and an inflammatory syndrome (erythrocyte sedimentation rate $100 \mathrm{~mm} / 1 \mathrm{st}$ hour, C-reactive protein 52 $\mathrm{mg} / \mathrm{l})$. Calcemia, phosphatemiaand liver function were normal. Angiotensin-converting enzyme assay was normal. Urinary analysis revealed proteinuria (0.9g/24 hours) and sterile pyuria. Viral, fungal and bacterial investigations (cytomegalovirus, Epstein-Barr virus, hepatitis B and $\mathrm{C}$ virus, syphilis, toxoplasmosis and tuberculosis) were negative. Rheumatoid factor, anti-nuclear antibodies and HLA-B27 were negative. 
Chest radiography was normal. Salivary gland biopsy was normal. Renal ultrasound showed normal echogenicity and morphology. Kidney biopsy revealed chronic active interstitial nephritis with infiltration by mononuclear cells and mild fibrosis (Fig. 1). Granulomas were absent. Glomeruli and tubular structures were normal. Immunofluorescence showed positive linear staining of the tubular basement membrane for C3, IgG, IgM and IgA.

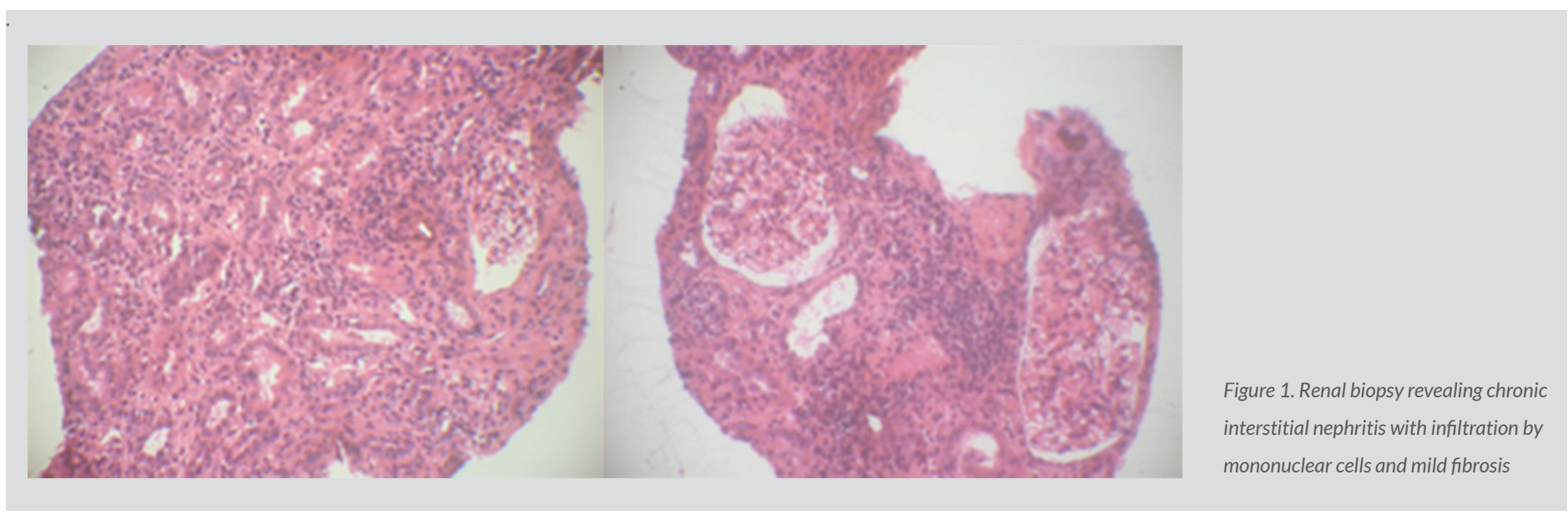

TINU syndrome was diagnosed. The patient was started on pulses of methyl prednisolone; high doses of prednisone were administered for 6 weeks and then tapered to $10 \mathrm{mg}$ a day. This treatment was accompanied by ocular corticosteroid and mydriatic eye drops. By the end of this regimen, renal function had improved (creatinine $73 \mu \mathrm{mol} / \mathrm{l}$ ) and uveitis had resolved.

Two years later, ocular redness and pain recurredand anterior uveitis relapse was diagnosed. The patient experienced joint pain. Physical examination revealed asymmetrical blood pressure $(120 / 70 \mathrm{mmHg}$ in the right arm and 100/60 $\mathrm{mmHg}$ in the left arm) and a decreased left brachial artery pulse. The patient was asymptomatic. Inflammatory markers were positive and includedan elevated erythrocyte sedimentation rate (140 mm/1st hour) and C-reactive protein levels (33 mg/dl). Renal function was normal. Ultrasound with colour Doppler identified homogeneous and echogenic wall thickening of the left common carotid artery. Computed tomography revealed bilateral circumferential and regular wall thickening of the internal carotid arteries, predominantlyof the right artery (Fig. 2).

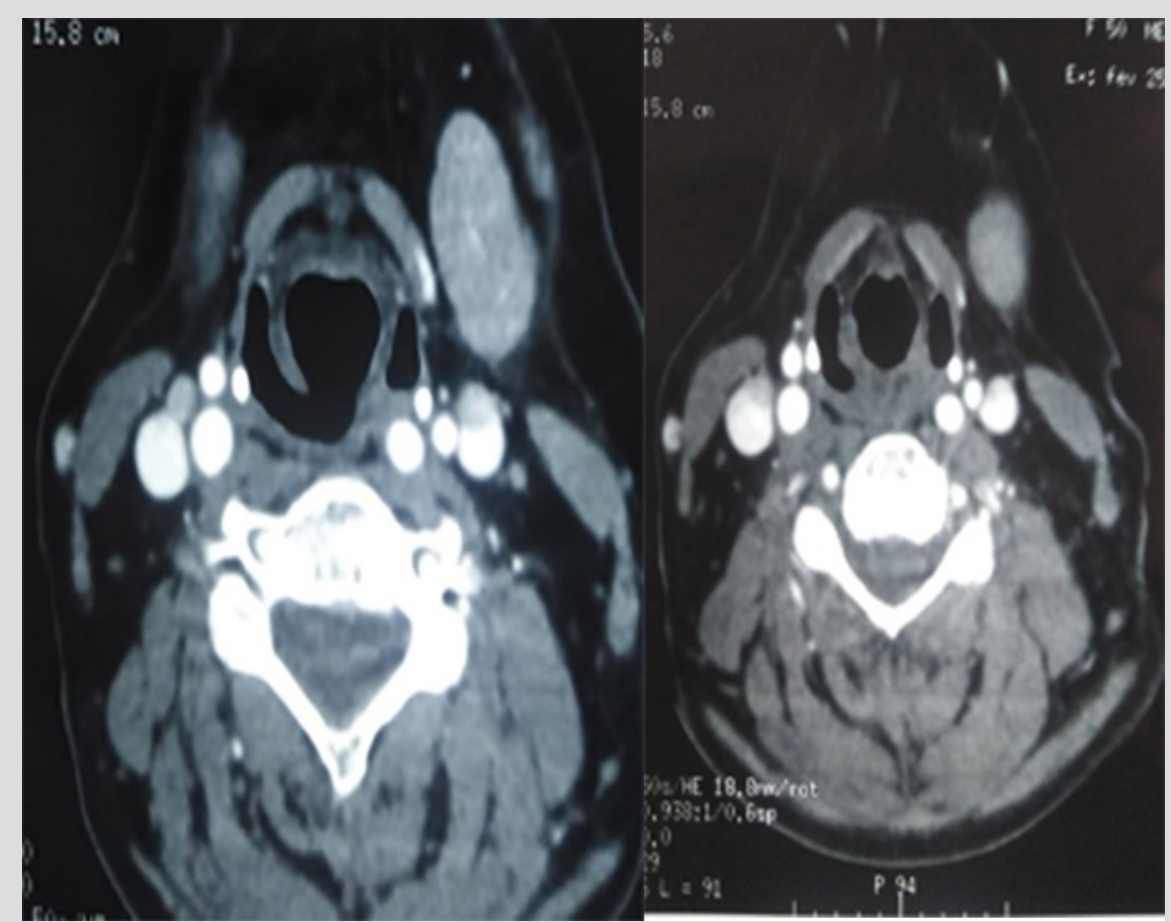

Figure 2. Computed tomography revealing bilateral circumferential and regular wall thickening of the internal carotid arteries 
TA was diagnosed. Anterior granulomatosis uveitis was identified without kidney disorder. High doses of oral prednisone (1mg/kg/day) accompanied by anti-platelet therapy were started. Inflammatory parameters normalized within 6 weeks. Prednisone was gradually reduced to a maintenance dose of $10 \mathrm{mg} /$ day. At follow-up, 6 years later, the patient was still asymptomatic and computed tomography assessment was unremarkable.

\section{DISCUSSION}

TINU syndrome is defined asacute interstitial nephritis associated with uveitis in the absence of systemic disease that can cause either interstitial nephritis or uveitis ${ }^{[5]}$. It manifests with non-specific signs and specific symptoms of renal disease and ocular involvement. The diagnostic criteria for TINU syndrome were described in 2001 by Mandeville et al. ${ }^{[5]}$. Clinical features of ocular disease are less defined than those of renal disorder. Bilateral anterior granulomatous uveitis can occur with or without intermediate or posterior uveitis. Ocular disease was bilateral in $77 \%$ of cases and unilateral in $23 \%$.

Our patient had anterior unilateral granulomatous uveitis. Her diagnosis of renal disorder was based on abnormal renal function findings, abnormal urine analysis results (low-grade proteinuria, urinary eosinophils, pyuria or haematuria without infection, increased beta- 2 microglobulin), and systemic illness lasting more than 2 weeks associated with histopathological findings of tubulointerstitial nephritis. Renal failure and abnormal urinary analysis suggested a kidney biopsy, which revealed active interstitial nephritis. Uveitis is diagnosed after interstitial nephritis in $65 \%$ of patients, before interstitial nephritis in $21 \%$ and at the same time in $15 \%{ }^{[5]}$. In our case, ocular manifestations and renal disorder developed simultaneously. Ocular prognosis appears to be good in such patients. Persistent renal dysfunction was described in $11 \%$ of cases ${ }^{[5]}$. Recurrence of uveitis in TINU syndrome has been frequently reported (56\% of patients) ${ }^{[5]}$. Relapse can occur up to 2 years after the initial uveitis ${ }^{[5]}$, which was the case in our patientwho represented after 2.5 years without renal disorder.

TAis a granulomatous vasculitis affecting largevessels and causinggeneralized and vascular symptoms. Its manifestations range from impalpable pulses or arterial bruits in an asymptomatic patient to life-threatening neurological manifestations. The diagnosis is based on the diagnostic criteria of Ishikawa modified by Sharma et al. ${ }^{[6]}$. In our patient, the diagnosis of TA was suspected in light of asymmetrical blood pressure, a decreased left brachial artery pulse and high inflammatory markers, and confirmed by imaging findings. However, we couldnot conclude definitely that the uveitis was caused by TINU syndromeas in this context it could be an uncommon manifestation of TA.Uveitis was first reported as an ocular manifestation of TAby Stone et al. ${ }^{[7]}$. The more common ocular manifestation of TA include episcleritis and Takayasu's retinopathy (four stages) ${ }^{[8-13]}$. Corticosteroids are effective for both uveitis and TA.

\section{CONCLUSION}

In our case, TA was discovered incidentally in a patient followed for TINU syndrome, demonstrating thatrare autoimmune diseases can coexist. Consequently, thorough physical examination and investigation is very important. To our knowledgethis is the first such case described in the literature.

\section{REFERENCES}

1. Vaideeswar P, Deshpande J. Pathology of Takayasu arteritis: a brief review. Ann Ped Cardiol 2013;6(1):52-58.

2. Korkmaz C, Zubaroğlu I, Kaya T, AkayOM.Takayasu's arteritis associated with rheumatoid arthritis: a case report and review of the literature. Rheumatology (Oxford) 2001;40(12):1420-1422.

3. Mirfeizi Z, HashemzadehK, Fazlinejad A.Association of ankylosing spondylitis and Takayasu's arteritis: a case report. Journal of Case Reports in Practice 2015;3(3).

4. Bulum J, Car N, Smircic-Duvnjak L, Gracin S, Metelko Z. Takayasu's arteritis and chronic autoimmune thyroiditis in a patient with type 1 diabetes mellitus. Clin Rheumatol 2005;24(2):169-171.

5. Mandeville JTH, Levinson RD, Holland GN. The tubulointerstitial nephritis and uveitis syndrome. Surv Ophthalmol 2001:46(3):195-208.

6. Sharma BK, Jain S, Suri S, Numano F. Diagnostic criteria for Takayasu arteritis in India. Heart Vessels 1992;7(Suppl):37-43.

7. Stone JH, NousariHC, Jabs DA, Griffith LS. Takayasu's arteritis associated to anterior uveitis and cutaneous extravascular necrotizing granuloma. J Clin Rheumatol 1998;4(6):319-322.

8. Arnaud L, Haroche J, Piette J-C, Amoura Z. L'artérite de Takayasu : mise au point à propos d'une série monocentrique de 82 patients. Rev Med Interne 2010;31(3):208-215.

9. Nithyanandam S, Mohan A, Sheth U. Anterior ischemic optic neuropathy in a case of Takayasu's arteritis. Oman J Ophthalmol 2010;3(2):94-95.

10. Chun SY, Park S-J, Park IK, Chung H, Lee J. The clinical and ocular manifestations of Takayasu arteritis.Retina 2001;21(2):132-140.

11. Peter J,David S, Joseph G, Horo S, Danda D.Hypoperfusive and hypertensive ocular manifestations in Takayasu arteritis. Clin Ophthalmol $2010 ; 4: 1173-1176$.

12. Vaideeswar P, Deshpande J. Pathology of Takayasu arteritis: a brief review. Ann Pediatr Cardiol 2013;6(1):52-58.

13. Loukil I, Mallouch N, Hachicha F, Bhiri R, Hijazi A, Jeddi Blouza A.[Ischemic retinopathy in Takayasu disease]. PresseMed 2012;41(12 Pt 1):e594-598. 